\title{
MODELAGEM DA PERDA DE CARGA EM TUBULAÇÕES COMERCIAIS, CONDUZINDO ÁGUA RESIDUÁRIA
}

\author{
José Antonio Rodrigues de Souza ${ }^{1}$, Débora Astoni Moreira ${ }^{2}$, Wilson Denículi ${ }^{3}$
}

Objetivou-se, neste trabalho, modelar a perda de carga contínua, em tubulações comerciais de aço zincado, utilizados na condução da água residuária de avicultura (ARA). A água residuária foi avaliada em seis concentrações de sólidos totais $\left(0,26 ; 0,46 ; 0,73 ; 0,94 ; 1,63\right.$ e 2,89 dag $\left.\mathrm{L}^{-1}\right)$, em tubulações comerciais de aço zincado, nos diâmetros comerciais de 50 a $150 \mathrm{~mm}$. De acordo com os resultados, verificou-se que as equações empíricas, ajustadas com a aplicação das metodologias de Sampaio et al., Duffy \& Titchener e Hazen-Williams modificada, apresentaram bons ajustes, com coeficiente de correlação acima de 0,90 , sendo recomendada sua aplicação no dimensionamento de sistemas de bombeamento dessas águas residuárias.

Palavras-Chave: resíduos agropecuários, bombeamento, hidráulica.

\section{ABSTRACT \\ MODELLING OF LOAD LOSS IN COMMERCIAL PIPES TRANSPORTING WASTEWATER}

This objective of this study was to model the continuous load loss in the commercial zinced steel pipes used in the management of wastewater from a poultry shed (WP). The WP was evaluated at six concentrations of the total solids of $0.26,0.46,0.73 ; 0.94,1.63$ or $2.89 \mathrm{dag} \mathrm{L}^{-1}$ was transported using commercial zinced steel pipes of 50 to $150 \mathrm{~mm}$ diameters. The empirical equations adjusted with the methodologies of Sampaio et al., Duffy \& Titchener or modified Hazen-Williams gave good results, with correlation coefficient above 0.90 and its application is recommended to design projects.

Keywords: agricultural waste, pumping, hydraulic.

\section{Recebido para publicação em 24/08/2009. Aprovado em 13/01/2011.}

1- Eng. Agrícola, Prof. IF Goiano - Campus Urutaí, Rodovia Geraldo da Silva Nascimento, km 2,5, Urutaí-GO, email: jarstec@yahoo.com.br;

2- Bacharel em Química, Profa UEMG unidade Ubá, Viçosa-MG, email: deboraastoni@yahoo.com.br;

3- Eng. Agrônomo, Prof UFV, Viçosa-MG, email: deniculi@ufb.br. 


\section{INTRODUÇÃO}

A utilização de águas residuárias, na agricultura, é uma alternativa para controle da poluição das águas superficiais e subterrâneas, além da disponibilização de água e fertilizantes para as culturas, reciclagem de nutrientes e aumento na produção agrícola. Entretanto, para que isso possa se tornar uma prática viável, é preciso que sejam desenvolvidas técnicas de tratamento, aplicação e manejo de águas residuárias.

Sabe-se que a fertirrigação, quando realizada com águas residuárias de avicultura, opera com características hidráulicas praticamente desconhecidas, podendo levar técnicos a cometerem erros consideráveis no seu dimensionamento.

Essa diferença pode ser comprovada nos trabalhos desenvolvidos por Zinato et al (2007), que classificaram reologicamente água residuária de avicultura como Plástico de Bigham e Souza (2005), que obteve comportamento hidráulico diferente para diversas concentrações de sólidos na água residuária de avicultura, em relação à água limpa.

Existem diversas fórmulas para estimar a perda de carga contínua, em condutos forçados com seção circular e área constante. De acordo com Duffy (1976), os métodos de avaliação dessa perda de carga está agrupada em três categorias: correlações gráficas, equações empíricas e equações que possibilitam a estimativa do fator de atrito, sendo as duas últimas as mais utilizadas e de maior precisão, pela facilidade de acesso ao computador.

Dentre as equações que utilizam o fator de atrito, a mais usada é a Equação Universal, ou seja:

$J=\frac{f}{D} \frac{V^{2}}{2 g}=\frac{8 f}{D^{5}} \frac{Q^{2}}{\pi^{2} g}$

em que

$\mathrm{J}=$ perda de carga unitária $\left(\mathrm{m} \mathrm{m}^{-1}\right)$;

$\mathrm{f}=$ fator de atrito (adimensional);

$\mathrm{D}=$ diâmetro interno da tubulação (m);

$\mathrm{V}=$ velocidade média do fluido $\left(\mathrm{m} \mathrm{s}^{-1}\right)$;

$\mathrm{g}=$ aceleração da gravidade $\left(\mathrm{m} \mathrm{s}^{-2}\right) ; \mathrm{e}$

$\mathrm{Q}=$ vazão média do fluido $\left(\mathrm{m}^{3} \mathrm{~s}^{-1}\right)$.

Existem várias equações que permitem estimar o fator de atrito para condutos circulares de seção constante, em função da rugosidade relativa das paredes da tubulação e do número de Reynolds. Sampaio (1999), por meio da Equação 2, apresentou o modelo de Lee \& Duffy (1976) modificado, obtendo resultados estatisticamente satisfatórios.

$$
\frac{1}{\sqrt{f}}=a(\ln \text { Rey })(\sqrt{f})+b
$$

em que

$\mathrm{f}=$ fator de atrito (adimensional);

Rey $=$ número de Reynolds (adimensional); e $\mathrm{a}$ e $\mathrm{b}=$ constantes características do fluido (adimensionais).

Uma equação empírica, que estima a perda de carga para fluidos não newtonianos, foi desenvolvida por pesquisadores atuantes nas áreas de alimentos, papel e celulose e de transporte de óleo em refinarias (DUFFY \& TITCHENER, 1974).

$J=\beta_{1} V^{\beta_{2}} S T^{\beta_{3}} D^{\beta_{4}}$

em que

$\mathrm{J}=$ perda de carga unitária $\left(\mathrm{m} \mathrm{m}^{-1}\right)$;

$\mathrm{V}=$ velocidade média $\left(\mathrm{m} \mathrm{s}^{-1}\right)$;

$\mathrm{D}=$ diâmetro interno da tubulação (m);

$\mathrm{ST}=$ concentração de sólidos totais $\left(\operatorname{dag} \mathrm{L}^{-1}\right) ; \mathrm{e}$

$\beta_{1}, \beta_{2}, \beta_{3}$ e $\beta_{4}=$ constantes características do fluido (adimensionais).

As equações empíricas usadas no cálculo da perda de carga apresentam, em geral, melhores resultados do que os métodos de correlações gráficas e as equações que estimam o fator de atrito (DUFFY, 1976).

Sampaio et al. (2000 e 2001), na busca de modelos matemáticos que estimassem a perda de carga distribuída em tubulações de aço zincado, ferro galvanizado e PVC, com diâmetros comerciais de 2 a 6 polegadas, utilizando como fluidos circulantes as águas residuárias de suinocultura e bovinocultura, com diferentes concentrações de sólidos totais, apresentaram a Equação 4 , como uma proposta de modificação do modelo de HazenWilliams. 
$J=\frac{k 1(S T)^{k 2} Q^{k 3}}{C^{k 4} D^{k 5}}$

em que

$\mathrm{J}=$ perda de carga unitária $\left(\mathrm{m} \mathrm{m}^{-1}\right)$;

$\mathrm{ST}=$ concentração de sólidos totais $\left(\operatorname{dag} \mathrm{L}^{-1}\right)$;

$\mathrm{Q}=$ - vazão média do fluido $\left(\mathrm{m}^{3} \mathrm{~s}^{-1}\right)$;

$\mathrm{C}=$ coeficiente de rugosidade (adimensional);

$\mathrm{D}=$ diâmetro interno da tubulação $(\mathrm{m}), \mathrm{e}$

k1, k2, k3, k4 e k5 = parâmetros de ajuste da equação (adimensionais).

Sampaio et al. (2000 e 2001) concluíram que os métodos que estimam a perda de carga baseados na equação universal não apresentaram bons ajustes, principalmente, quando se utilizou o número de Reynolds generalizado. Segundo os autores, as equações empíricas obtidas, utilizando-se as metodologias de Duffy \& Titchener (1974) e Hazen-Williams modificada, para as águas residuárias de bovinocultura e de suinocultura, que relacionam a perda de carga com vazão, diâmetro, coeficiente de rugosidade e concentração de sólidos totais, apresentaram bons resultados, com coeficientes de determinação superiores a $98 \%$, podendo-se recomendar sua utilização para dimensionamento de projetos hidráulicos pressurizados, para escoamento das duas águas residuárias.

Neste trabalho, teve-se por objetivo ajustar equações de perda de carga contínua para tubulações de aço zincado, nos diâmetros comerciais de 50 a $150 \mathrm{~mm}$, conduzindo água limpa e água residuária de avicultura (ARA).

\section{MATERIAIS E MÉTODOS}

O experimento foi conduzido na Área Experimental de Hidráulica, Irrigação e Drenagem, do Departamento de Engenharia Agrícola, da Universidade Federal de Viçosa, em Viçosa, Minas Gerais.

$\mathrm{Na}$ Figura 1, está apresentado o esquema da bancada experimental, com os conjuntos motobombas, as tubulações, os piezômetros e os detalhes internos dos reservatórios.

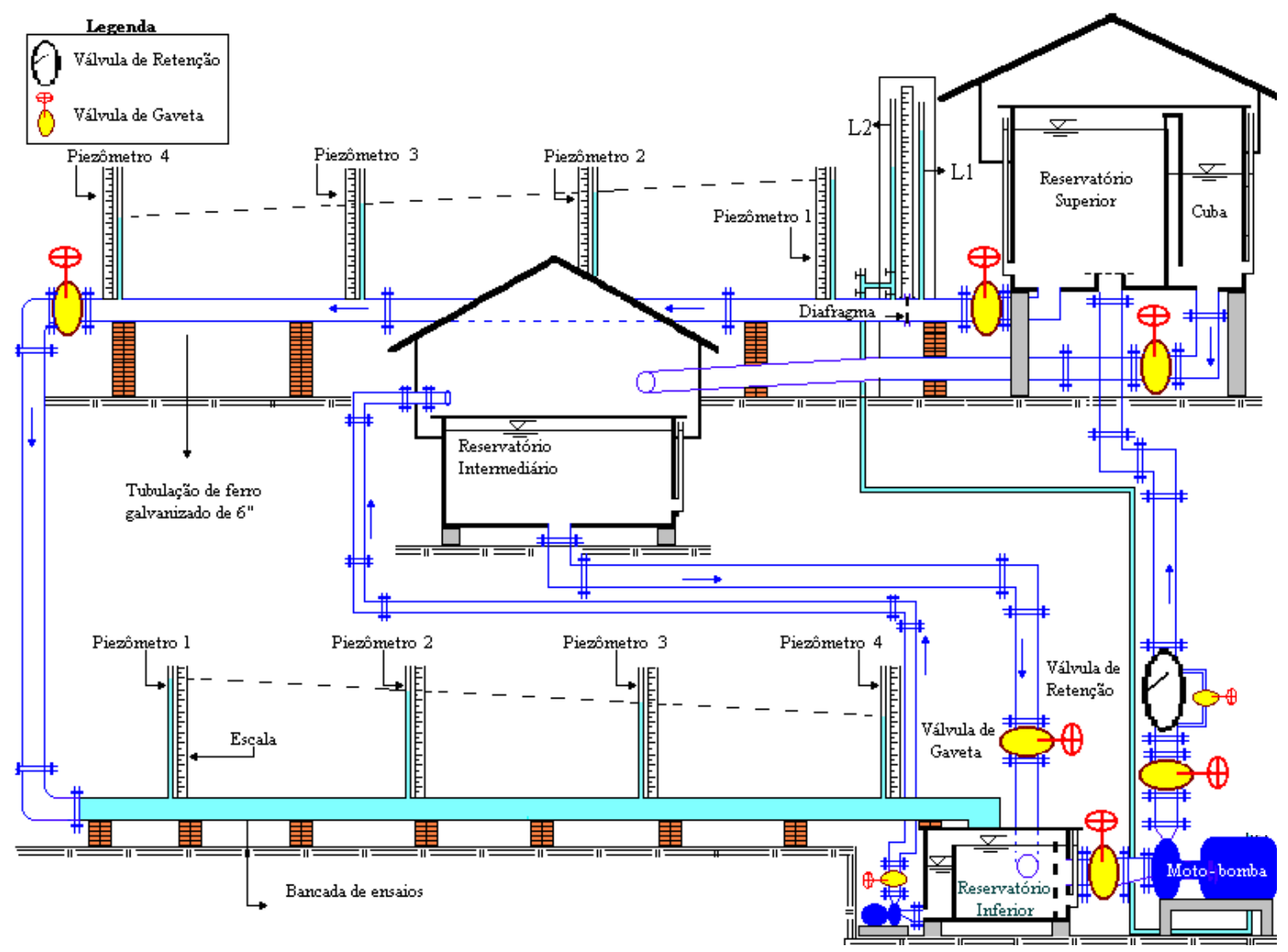

Figura 1. Esquema representando a bancada experimental. 
Foram utilizadas tubulações de aço zincado, nos diâmetros internos de 73,54; 99,83; 125,24 e $150,43 \mathrm{~mm}$. A perda de carga foi medida num trecho com $18 \mathrm{~m}$ de comprimento, por meio de 4 piezômetros espaçados de 6 metros, resultando em três trechos de 6 metros.

A vazão foi medida por meio de diafragma previamente calibrado, instalado na tubulação derivada do reservatório superior. A fim de garantir a condução dos ensaios com as tubulações cheias de fluido, instalaram-se curvas de $90^{\circ}$, voltadas para cima, na extremidade final de cada tubulação.

As tubulações avaliadas eram conectadas à tubulação de ferro galvanizado, de 155,58 mm de diâmetro, por meio de reduções concêntricas flangeadas, sendo colocadas em nível, de modo a permitir o uso da Equação 1 na determinação do fator de atrito.

Utilizou-se como fluido circulante a água limpa e ARA, em seis diferentes concentrações de sólidos totais $(0,26 ; 0,46 ; 0,73 ; 0,94 ; 1,63 \mathrm{e}$ 2,89 dag $\mathrm{L}^{-1}$ ), obtidas mediante adição e mistura de esterco de galinhas poedeiras, em reservatório de $10 \mathrm{~m}^{3}$ contendo água.

Primeiramente, o esterco foi moído e passado em peneira, com abertura de $6 \mathrm{~mm}$, para a retirada de penas e outros materiais indesejáveis, sendo, posteriormente, adicionado ao reservatório com o fluido em circulação, para evitar sedimentação e facilitar a homogeneização. Após a adição de esterco correspondente a cada concentração, o efluente circulou durante cinco horas, depois de ficar pelo menos 12 horas em repouso para absorção de água.

A ração das galinhas poedeiras do galpão, onde foi obtido o esterco, continha cerca de $60 \%$ de milho, $25 \%$ de farelo de soja, $7 \%$ de calcário e $2 \%$ de fosfato bicálcico, além de suplementos minerais, óleos e aditivos, totalizando $6 \%$.

Os ensaios foram conduzidos da seguinte maneira: o fluido era bombeado do reservatório inferior para o reservatório superior, de onde parte do fluido escoava por gravidade para as tubulações situadas na bancada de ensaios, que apresentava um desnível de $4,5 \mathrm{~m}$ em relação ao nível constante do reservatório superior. Em seguida, o fluido era descarregado no reservatório inferior. $\mathrm{O}$ excedente do fluido do reservatório superior era conduzido para o reservatório intermediário para, posteriormente, ser conduzido para o reservatório inferior, onde era novamente bombeado, fechando-se, dessa maneira, o ciclo.

Instalou-se no interior do reservatório inferior uma chapa sem perfuração, para estabelecer nível constante do fluido, e uma chapa perfurada, para reduzir a turbulência causada pela entrada de fluido proveniente da tubulação avaliada. O excesso de fluido neste reservatório era recebido num compartimento, localizado à esquerda da chapa metálica sem perfurações, sendo, em seguida, bombeado para o reservatório intermediário, com um conjunto motobomba auxiliar dotado de chaveboia, que efetuava acionamento e desligamento automáticos desse recalque, toda vez que o nível atingia a altura de $0,67 \mathrm{~m}$ em relação ao fundo.

No reservatório superior, foi instalada uma chapa metálica circular, posicionada horizontalmente nas proximidades da seção de entrada, a $25 \mathrm{~cm}$ do fundo, para reduzir a turbulência do fluido. Outra chapa metálica com entalhe em " $V$ " foi instalada na parte superior, semelhante a um vertedor triangular, mantendose o nível constante do fluido, para condicionar todos os ensaios às mesmas características de escoamento e altura de carga. Possibilitou-se, também, a criação de um compartimento (cuba) para uso na calibração do diafragma.

De posse dos dados de perda de carga, concentração de sólidos totais, vazão e diâmetros de tubulação, avaliaram-se os modelos de estimativa de perda de carga de Sampaio et al (1999), Duffy \& Titchener (1974) e HazenWilliams modificado, utilizando-se o programa estatístico SAEG.

\section{RESULTADOS E DISCUSSÃO}

No Quadro 1, são apresentados os resultados das análises física e química da água limpa e da água residuária de avicultura. Neste quadro, observa-se que os valores de sólidos sedimentáveis e de peso específico da ARA aumentaram com a 
concentração de sólidos totais.

Utilizando-se a Equação 1 para o cálculo do fator $\mathrm{f}$, verificou-se que, para uma mesma vazão e diâmetro da tubulação, o valor do fator $\mathrm{f}$ diminuiu em relação à água limpa até a concentração de sólidos totais de 0,46 dag L $^{-1}$, e aumentou, da concentração de 0,46 dag $\mathrm{L}^{-1}$ à 2,89 dag $\mathrm{L}^{-1}$. Tagliaferre (2003), estudando perda de carga em tubos de polietileno, conduzindo água residuária de suinocultura, obteve comportamento semelhante, encontrando perda de carga menor que a da água limpa, em concentrações de sólidos totais que variaram de 1,15 a $1,75 \mathrm{dag}^{-1}$.

$\mathrm{Na}$ Figura 2, são apresentadas as curvas ajustadas, relacionando perda de carga unitária (J) em função da vazão (Q), para água limpa e seis concentrações de ARA, nos cinco diâmetros avaliados.

Pode-se verificar, na Figura 2, que a maior e a menor perda de carga sempre ocorreram, para água limpa e ARA, na concentração de 0,46 dag L $^{-1}$, respectivamente. Associando-se os resultados da perda de carga com a viscosidade nas várias concentrações de ARA, verificase uma tendência de que a perda de carga seja menor em fluidos de viscosidade menor que a da água limpa. Com menor viscosidade, menos energia será dissipada por atrito, implicando menor perda de carga.

Segundo Azevedo Netto et al. (1998), no escoamento em tubos, mesmoemregime turbulento liso, sempre existe uma camada laminar junto às paredes. Esta camada, em algumas situações de escoamento, cobre boa parte da rugosidade das tubulações ensaiadas, diminuindo o atrito com o fluido, contribuindo para a redução na perda de carga em relação à água limpa.
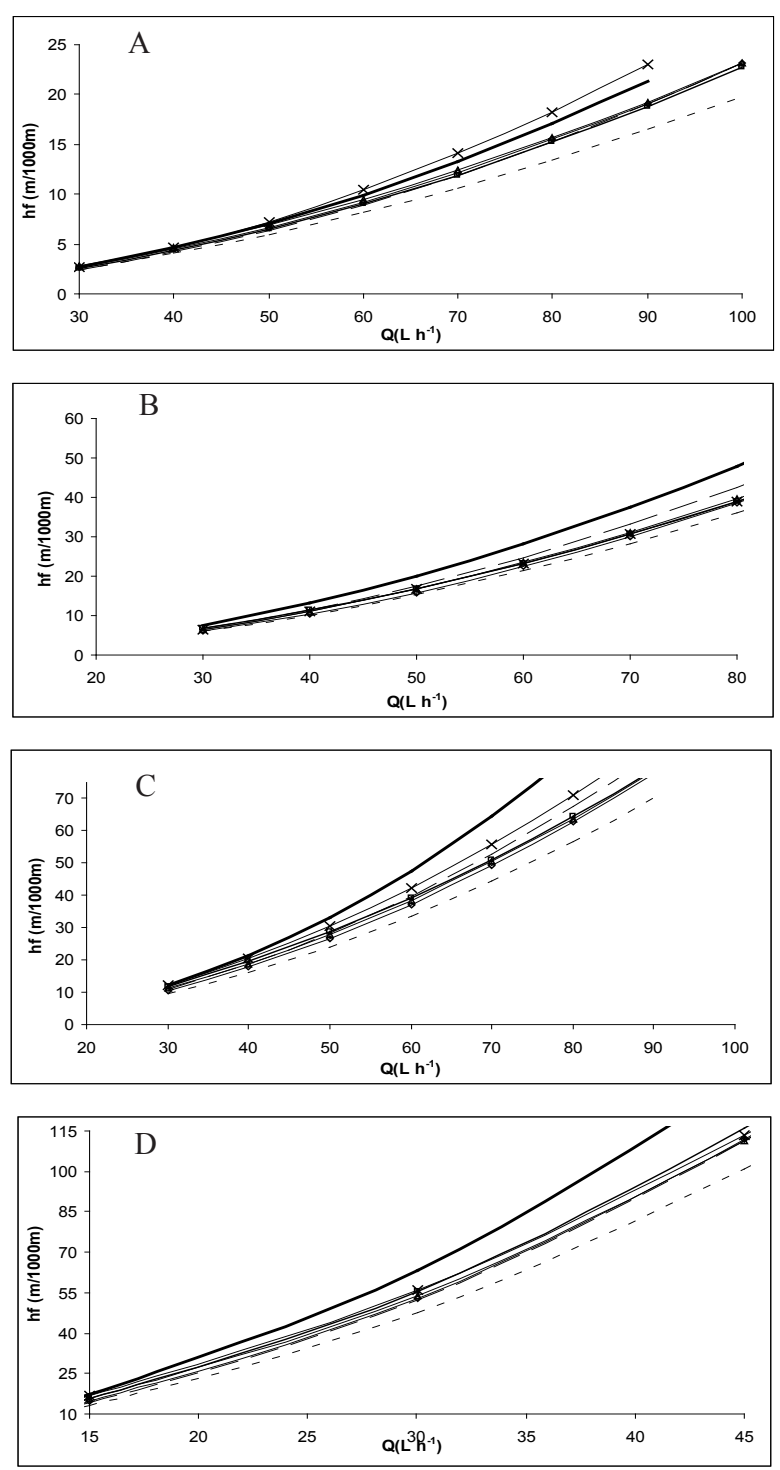

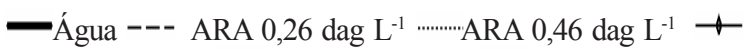
ARA 0,73 dag L-1 ARA 0,94 dag L ${ }^{-1}$ ARA 1,64 dag L L $^{-1}$ * ARA 2,89 dag L $\mathrm{L}^{-1}$

Figura 2. Curvas ajustadas, relacionando perda de carga unitária $(\mathrm{J}-\mathrm{m} / 1000 \mathrm{~m})$, em função da vazão $\left(\mathrm{Q}-\mathrm{m}^{3} \mathrm{~h}^{-1}\right)$, para a tubulação de aço zincado nos diâmetros de (A) $150,43 \mathrm{~mm}$; (B) $125,24 \mathrm{~mm}$; (C) $99,83 \mathrm{~mm}$ e (D) $73,54 \mathrm{~mm}$.

Quadro 1. Valores das características física e química da água limpa e água residuária de avicultura (ARA)

\begin{tabular}{c|c|cccccc}
\hline & Água & \multicolumn{7}{|c}{ ARA } \\
\hline $\mathrm{ST}\left(\mathrm{dag} \mathrm{L}^{-1}\right)$ & 0,01 & 0,26 & 0,46 & 0,73 & 0,94 & 1,63 & 2,89 \\
$\mathrm{SD}\left(\mathrm{mL} \mathrm{L}^{-1}\right)$ & $<0,1$ & 14 & 27 & 52 & 81 & 173 & 297 \\
$\gamma\left(\mathrm{kgf} \mathrm{m}^{-3}\right)$ & 999,48 & 1.003 & 1.004 & 1.006 & 1.004 & 1.009 & 1.015 \\
$\eta\left(\mathrm{Pa} \mathrm{s}^{2}\right)$ & 0,00130 & 0,000836 & 0,0007822 & 0,00085540 & 0,0002796 & 0,0007191 & 0,0002706 \\
$\mathrm{pH}$ & 7,05 & 7,47 & 7,19 & 7,28 & 7,33 & 7,12 & 7,17 \\
\hline
\end{tabular}

ST $=$ sólidos totais; $\mathrm{SD}=$ sólidos sedimentáveis; $\gamma=$ peso específico; $\eta=$ viscosidade aparente; $\mathrm{e}$ $\mathrm{pH}=$ potencial hidrogeniônico. 
Quadro 2. Equações ajustadas do fator de atrito (f) e perda de carga (J) para as diferentes concentrações de ARA avaliadas

\begin{tabular}{lcc}
\hline \multicolumn{1}{c}{ Modelo } & Equação ajustada & $\mathbf{R}^{2}$ \\
\hline Sampaio et al (1999) & $\frac{1}{\sqrt{f}}=-3,2814 S T^{-0,0278}(\operatorname{In}$ Rey $) \sqrt{f}+13,0463$ & 0,9040 \\
Duffy e Titchener (1974) & $\mathrm{J}=0,003714 \mathrm{~V}^{1,71039} \${ }^{0,0182} \mathrm{D}^{-0,5538}$ & 0,9547 \\
Hazen-Williamns modificado & $\mathrm{J}=\frac{10,649 \mathbf{Q}^{1,8518}}{\mathrm{C}^{1,8523} \mathrm{D}^{4,8712}}$ & 0,9990 \\
\hline
\end{tabular}

No Quadro 2, são apresentados os resultados dos modelos de estimativa de perda de carga contínua, baseados nas equações de HazenWilliams e Universal, após teste de identidade de modelo, para determinar a equação comum para os diferentes diâmetros e concentrações de ARA.

em que

$\mathrm{f}=$ fator de atrito (adimensional);

$\mathrm{ST}=$ sólidos totais $\left(\operatorname{dag} \mathrm{L}^{-1}\right)$;

Rey = número de Reynolds (adimensional);

$\mathrm{V}=$ velocidade média do fluido $\left(\mathrm{m} \mathrm{s}^{-1}\right)$;

$\mathrm{D}=$ diâmetro da tubulação $(\mathrm{m})$;

$\mathrm{Q}=\operatorname{Vazão}\left(\mathrm{m}^{3} \mathrm{~s}^{-1}\right)$; e

$\mathrm{C}=$ coeficiente de rugosidade (adimensional).

No ajuste das equações pelo modelo de Sampaio et al. (1999), todas as observações se processaram no regime turbulento, com número de Reynolds mínimo em torno de 100.000. Como a viscosidade do fluido era conhecida, ela foi utilizada para determinar o número de Reynolds.

Nas equações encontradas, para o modelo matemático de Duffy \& Titchener (1974), o expoente da velocidade apresenta-se dentro da faixa apresentada por Duffy (1976), para diferentes fluidos escoantes não newtonianos, e aproxima-se daqueles apresentados por Hazen \& Williams (1963). O baixo valor dos expoentes da concentração de sólidos totais é um indicativo de que o efeito desta variável na perda de carga foi pequeno, para as concentrações estudadas.

As estimativas de perda de carga que mais se aproximaram dos dados observados em campo foram correspondentes às equações de melhor ajuste, destacando-se os modelos de Duffy \& Titchener (1974) e Hazen \& Williams modificado, sendo que, com a utlitização deste último, podese estimar a perda de carga mais próxima dos valores encontrados nos ensaios, subestimando, em média, a perda de carga para quaisquer vazões, concentrações de sólidos totais ou diâmetros. Ele proporcionou, em geral, os menores erros médios e desvios padrão, justificando, portanto, seu melhor ajuste, se comparados com os demais.

A diferença entre a perda de carga da água e a da ARA, comparando-se os valores medidos nos ensaios e os estimados utilizando-se o modelo de Hazen-Williams modificado, foi subestimado em 32 a - 9,7\%, na tubulação de aço zincado de $99,83 \mathrm{~mm}$.

\section{CONCLUSÕES}

- As viscosidades da ARA, em todas as concentrações analisadas, apresentaram-se menores que a da água;

- A perda de carga proporcionada pela ARA, para as concentrações de sólidos totais estudadas, foi menor do que para a água limpa, diminuindo até a concentração de 0,46 dag $\mathrm{L}^{-1}$ e voltando a crescer até a concentração de 2,89 dag $\mathrm{L}^{-1}$; e 
- As equações empíricas propostas neste trabalho, utilizando-se as metodologias de Sampaio et al. (1999), Duffy \& Titchener (1974) e HazenWilliams modificada e que relacionam a perda de carga com a vazão, diâmetro, coeficiente de rugosidade e concentração de sólidos totais, apresentaram bons resultados, com coeficientes de determinação acima de $90 \%$. Desta maneira, recomenda-se a sua utilização para dimensionamento de projetos, por serem equações simples e confiáveis.

\section{REFERÊNCIAS}

AZEVEDO NETTO, J.M., FERNADEZ, M.F., ARAÚJO, R., ITO, A.E. Manual de hidráulica $8^{\mathrm{a}}$ ed. São Paulo: Edgard Blucher, 670p, 1998.

DUFFY, G.G. A review and evolution of design methods for calculating friction loss in stock piping systems. Tappi, Atlanta, v.59, n.8, p.124-127, Aug, 1976.

DUFFY, G.G., TITCHENER, A.L. Design procedures for obtaining pipe friction los for chemical pulps. Tappi, Atlanta, v.57, n.5, p. 162166, may, 1974.

HAZEN, A., WILLIAMS, G. S. Hydraulic tables. New York: John Willey \& Sons, 1963. 120p.

SAMPAIO, S.C. Perda de carga em tubulações comerciais conduzindo águas residuárias de bovinocultura e suinocultura. 1999. 158f. Tese (Doutorado em Engenharia Agrícola) - Programa de Pós-Graduação em Engenharia Agrícola,
Universidade Federal de Viçosa, Viçosa, MG, 1999. SAMPAIO, S.C., DENICULI, W., OLIVEIRA, R.A., SILVA, D.D., CECON, P.R., PINTO, F.A. Equações de perda de carga distribuída para tubulações comerciais conduzindo água residuária de bovinocultura. Engenharia Agrícola. Jaboticabal, v.20, n.2, p.108-118, mai. 2000.

SAMPAIO, S.C., DENICULI, W., OLIVEIRA, R.A., SILVA, D. D., MATOS, A.T., MARTINEZ, M.A. Perda de carga contínua em tubulações conduzindo água residuária da suinocultura. Revista Brasileira de Engenharia Agrícola e Ambiental, v.5, n.3, p.391-396. 2001.

SOUZA, J.A.R. de. Desempenho de microaspersores operando com águas residuárias de avicultura e bovinocultura. 2005. 60f. Dissertação (Mestrado em Engenharia Agrícola) - Universidade Federal de Viçosa, Viçosa, MG, 2005.

TAgliaferre, C. Perda de carga em tubos de polietileno e entupimento de microaspersor operando com água residuária de suinocultura. Viçosa, MG: UFV, 2003. 75f. Dissertação(Mestrado em Engenharia Agrícola) - Universidade Federal de Viçosa, Viçosa, MG, 2003.

ZINATO, E.C; DENÍCULI, W.; SOUZA, J.A.R; BATISTA, R.O; MATOS, A.T.; OLIVEIRA, R.A; FERREIRA, D.C; SOARES, E.C. Caracterização Reológica De Água Residuária De Galinhas Poedeiras.Revista Engenharia na Agricultura, Viçosa, MG, v.15, n.2, 179-187, Abr./Jun., 2007. 